\title{
AGNs: DEMOGRAPHY AND REMNANTS
}

\author{
MARTIN J. REES \\ Institute of Astronomy \\ Madingley Road \\ Cambridge, CB3 0HA.
}

\section{Introduction}

In this contribution I shall first briefly review the arguments suggesting that most galaxies pass through a quasar or AGN phase early in their lifetime, perhaps concurrent with bulge formation. Then, as a footnote to the paper by van der Marel, I will discuss how relic black holes in quiescent galaxies could reveal themselves by the occasional flares resulting from tidal disruption of stars. In conclusion, I shall briefly discuss the phenomena associated with binary black holes, arguing that these are the likely outcome of galaxy mergers.

\section{Quasar Demography}

There is now firmer statistical evidence on the evolution of the quasar luminosity function at redshifts out to 4 . The highest comoving density occurs at between $z=2$ and $z=3.5$. Simple estimates then suggest that the comoving density of quasars is one or two per cent of the present comoving density of bright galaxies. Quantifying this further depends, of course, on what parts of the luminosity functions are compared. For a detailed discussion see Haehnelt and Rees (1993).

The rarity of quasars could imply that only a small fraction of galaxies experience a quasar phase, in which case the activity would need to last about $2 \times 10^{9}$ years. Alternatively, every galaxy could go through the phase, the quasar duration then needing to be only a few times $10^{7}$. Most recent discussions have favoured the second option, partly because there is a natural growth timescale for a black hole, of order $4 \times 10^{7}$ years, set by the accretion rate needed to yield an Eddington luminosity, and this timescale arises naturally in many specific models for the nuclear activity.

The origin of quasars is plainly linked to the early evolution of galaxies. In a recent discussion, Haehnelt and I considered this specifically in the context of a CDM model. However, the basic features of our discussion apply more generally. We argue that when big bulges form, a fraction of the baryons, some already processed through stars, condense 
into a massive central black hole. Typical high- $z$ quasars are supposed to be associated with this process, or its immediate aftermath, and have lifetimes of a few times $10^{7}$ years.

The accumulation of a central relativistic mass depends on the ability of a significant fraction of the galactic material to lose most of its angular momentum. The relevant material is likely to start off with sufficient angular momentum to provide rotational support at $\sim 100$ pc (Haehnelt and Rees 1993), but redistribution, allowing a substantial fraction to condense, is almost inevitable within $10^{8}$ years. There are indeed only two ways in which massive black hole formation could be avoided. One would be if the material in the central kiloparsec of a young galaxy condensed with essentially 100 per cent efficiency into stars that were all of low mass, so that no material remained gaseous; nor was any ejected and recycled. The second possibility, which seems equally unlikely, would be that gas in a self-gravitating disk could remain for at least 100 dynamical timescales without becoming subject to instabilities that would redistribute angular momentum. The various processes are depicted in Figure 1, reproduced from Rees (1993).

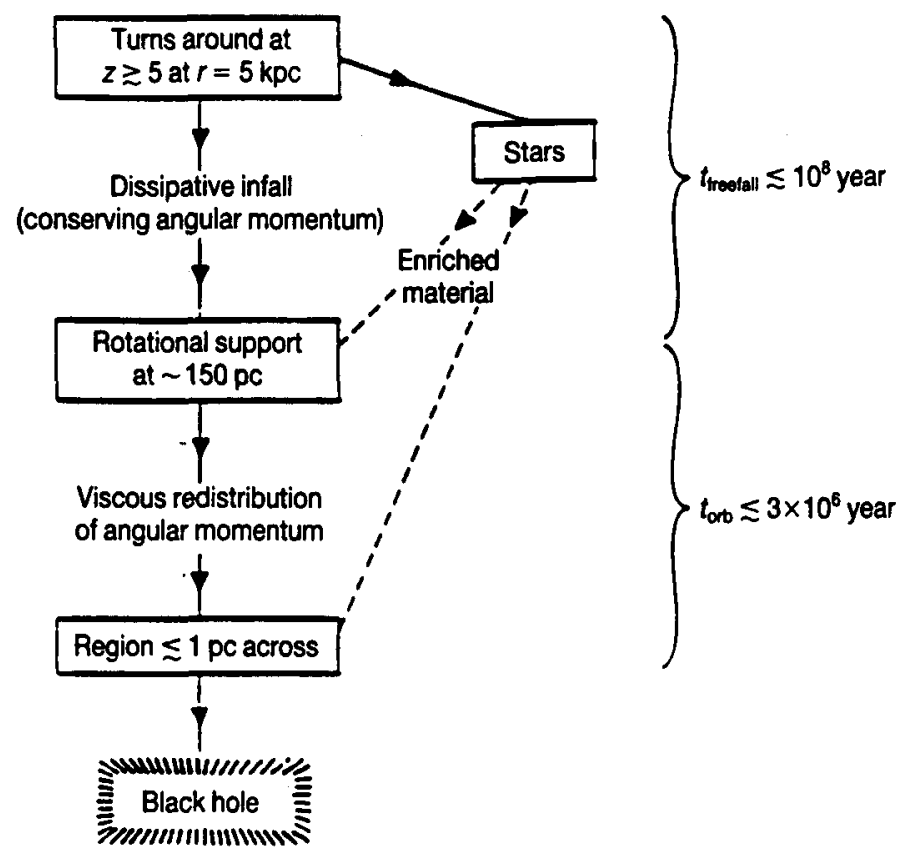

Fig. 1. Formation of the inner part of a galaxy. The outcome would be a stellar bulge surrounding a massive black hole. The fraction of the mass going into the hole depends on the rapidity with which angular momentum can be lost, on the efficiency of star formation, and on feedback effects from high-mass stars. Note that heavy elements can be synthesized within the period over which the black hole grows. From Rees (1993). 
The heavy elements in quasars could be produced within the $10^{8}$ years over which the bulge and central mass form. It would indeed not be surprising if the abundances reached more than solar levels, because the combination of high gas density and deep gravitational potential wells is optimal for retaining and recycling gas. This interpretation of high redshift quasars suggests that the phenomenon should be associated with a bright starburst, even though the latter would be unlikely to dominate the luminosity, for reasons which have been spelt out clearly by Heckman and others.

\section{Interaction of Stars with Quiescent Black Holes}

The growing evidence for massive black holes in the centres of nearby galaxies, reviewed by van der Marel in an accompanying paper, will eventually provide important constraints on ideas of quasar evolution. Obviously the mass distribution of remnants is related to some combination of quasar luminosity and lifetime during the early active phases. It will be interesting to know if the hole mass is correlated with the mass in the bulge. HST data should soon not only provide firmer estimates of central masses, but also more information on the star distribution in the surrounding cusp. This should allow more precise estimates of the rate at which stars diffuse on to sufficiently radial orbits to get tidally disrupted by the central hole. If the consequences of such disruption could be observed, this would provide an important consistency check on the massive black hole hypothesis, as well as opening up the possibility of new tests of strong gravity. The complex gas dynamics involved in stellar disruption, and the subsequent motion and possible swallowing of the debris, have been outlined elsewhere (Rees 1988, 1994; Kochanek 1994). There is general agreement that the actual disruption process does not cause conspicuous prompt radiation, but that a flare would be expected when the most tightly bound debris fell back towards the hole after moving in an eccentric orbit with a period of a few months. At much later times, the infall rate would decline as $t^{-\frac{5}{3}}$. Such an event could yield a flare where a quiescent galaxy would emit a quasar level luminosity for a period of months, or possibly one or two years. What is uncertain is the spectrum of the emergent radiation, and the efficiency with which it is produced. Quantifying this phenomenon is a computational challenge even to those with many gigaflops at their disposal. The phenomenon is inherently three-dimensional, and involves relativistic gas dynamics and radiative transfer with a large dynamic range.

These flares are nevertheless a robust prediction if black holes indeed exist in quiescent galaxies. We would not expect to see such an event in M31 in our lifetime, but a survey of the nearest few thousand galaxies should catch a few at the peak of a flare, and probably rather more in a state where the effects of the most recent tidal disruption are still discernible.

The amount of energy available, if the efficiency were as much as 10 per cent, would exceed by a thousand times the photon output of a supernova. The events would certainly, however, be varied and far from standardised. What we observe depends on the mass of the black hole, the nature of the star being swallowed, its minimum impact parameter, and also on the orientation of the orbit relative to our line of sight, and perhaps also relative to the spin axis of the hole.

In our own galactic centre, if there is indeed a black hole of a few million solar masses there, the expected disruption rate is about 1 per $10^{5}$ years. The time-averaged energy 
input from such events, both in kinetic form or in UV, could dominate other luminosities. Moreover, the debris ejected on hyperbolic orbits when the most recent disruption occurred could have some residual effect on physical conditions in the central parsec (Rees 1994).

In 1975 Chandrasekhar wrote: "In my entire scientific life ... the most shattering experience has been the realization that an exact solution of Einstein's equations of general relativity, discovered by the New Zealand mathematician Roy Kerr, provides the absolutely exact representation of untold numbers of massive black holes that populate the universe". It is now even clearer that there may indeed be black holes at the centres of most galaxies. But can we really "diagnose" whether they indeed have the properties that Einstein's equations predict?

The very large relativistic precession near black holes, as already mentioned, makes a qualitative difference to the fate of debris from disrupted stars. But in that context the effects are conflated with complicated gas dynamics; it will be a long time before this is well enough understood to provide quantitative tests. A more hopeful possibility would arise if a star could be captured, without disruption, into a relativistic orbit, which might modulate the output from an AGN. (Podsiadlowski and Rees 1994; Karas and Vokrouhlicky 1993 and references cited therein.)

\section{Supermassive Binaries}

There is another phenomenon where the effects of gravity and spacetime curvature are not confused by other physics: the coalescence of two massive black holes. Such events are inevitable. Most of the black holes in galaxies formed at redshifts of two or more, when quasar activity peaked. Moreover, most galaxies have experienced at least one merger since that time. When two galaxies merge, their central black holes spiral together under dynamical friction. When the holes get really close, gravitational radiation drives them towards coalescence. This is a grander version of the neutron star coalescence that LIGO may detect. However, the waves from supermassive binaries have a characteristic period of hours rather than a fraction of a second, and could be detected by space interferometers, such as the planned LISA, though the predicted event rate is discouragingly low (Haehnelt 1994). One astrophysical consequence of gravitational radiation is that there is a recoil in the final coalescence. A disconcerting possibility is then that some merged holes may have escaped from their host galaxy, and be now hurtling through intergalactic space.

\section{References}

Chandrasekhar, S. 1975, quoted in "Truth and Beauty" p54 (Chicago UP 1987).

Haehnelt, M. 1994, M.N.R.A.S., 269, 199.

Haehnelt, M. and Rees, M.J. 1993, M.N.R.A.S., 263, 168

Karas, V. and Vokrouhlicky, D. 1993, M.N.R.A.S., 265, 365.

Kochanek, C. 1994, Ap.J., 422, 508.

Podsiadlowski, P. and Rees, M.J., 1994, in "Evolution of X-ray Binaries", ed. S. Holt and C. Day, p403 (AIP Publications). 
Rees, M.J. 1988, Nature, 333, 523.

Rees, M.J. 1993, Proc. Nat. Acad. Sci., 90, 4840.

Rees, M.J. 1994, in "Nuclei of Nearby Galaxies", ed. R. Genzel and A. Harris (Springer, in press). 